\section{BRAZIULIAN JOURNAL \\ OF MEDICAL AND BIOLOGICAL RESF.ARCH}

www.bjournal.com.br
ISSN 0100-879X

Volume 42 (11) 993-1118 November 2009

BIOMEDICAL SCIENCES

AND

CLINICAL INVESTIGATION

Braz J Med Biol Res, November 2009, Volume 42(11) 993-1001

TMEM16 proteins: the long awaited calcium-activated chloride channels?

C.A. Flores, L.P. Cid, F.V. Sepúlveda and M.I. Niemeyer

The Brazilian Journal of Medical and Biological Research is partially financed by
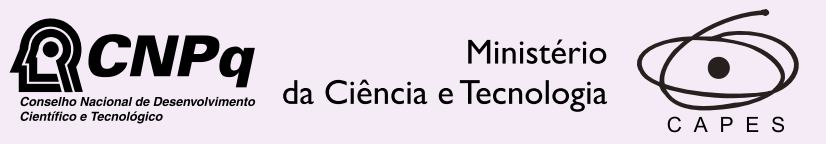

Ministério da Educação

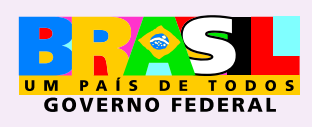

DFAPESP

Institutional Sponsors 


\title{
TMEM16 proteins: the long awaited calcium-activated chloride channels?
}

\author{
C.A. Flores ${ }^{1}$, L.P. Cid ${ }^{1}$, F.V. Sepúlveda ${ }^{1,2}$ \\ and M.I. Niemeyer ${ }^{1}$ \\ ${ }^{1}$ Centro de Estudios Científicos (CECS) and \\ ${ }^{2}$ Centro de Ingeniería de la Innovación del CECS (CIN), Valdivia, Chile
}

\begin{abstract}
Currents mediated by calcium-activated chloride channels (CaCCs), observed for the first time in Xenopus oocytes, have been recorded in many cells and tissues ranging from different types of neurons to epithelial and muscle cells. CaCCs play a role in the regulation of excitability in neurons including sensory receptors. In addition, they are crucial mediators of chloride movements in epithelial cells where their activity regulates electrolyte and fluid transport. The roles of CaCCs, particularly in epithelia, are briefly reviewed with emphasis on their function in secretory epithelia. The recent identification by three independent groups, using different strategies, of TMEM16A as the molecular counterpart of the CaCC is discussed. TMEM16A is part of a family that has 10 other members in mice. The discovery of the potential TMEM16 anion channel activity opens the way for the molecular investigation of the role of these anion channels in specific cells and in organ physiology and pathophysiology. The identification of TMEM16A protein as a CaCC chloride channel molecule represents a great triumph of scientific perseverance and ingenuity. The varied approaches used by the three independent research groups also augur well for the solidity of the discovery.
\end{abstract}

Key words: Calcium-activated chloride channels; TMEM16; ANO; Intestinal secretion; Salivary gland; Lacrimal gland

\section{Calcium-activated chloride conductance}

Currents mediated by calcium-activated chloride channels (CaCCs) were first observed in the early 1980's in Xenopus oocytes $(1,2)$ and salamander photoreceptors (3). Throughout the text we use the term CaCCs to describe macroscopic currents produced by calcium-activated chloride channels or to describe their single-channel activity recorded by the patch-clamp technique. More recently, they have been recorded in many cells and tissues ranging from different types of neurons to epithelial and muscle cells, as reviewed recently and comprehensively by Hartzell et al. (4).

There are at least two different functions that could be fulfilled by CaCCs in cells and tissues where they are expressed. First, by shifting the membrane potential CaCCs could have an effect on excitability in neurons including the modulation of transduction in sensory neurons. The effect could be one of stabilization of the membrane potential, if intracellular chloride concentration is close to thermodynamic equilibrium, or excitation or inhibition if intracellular chloride is above or below equilibrium, respectively. Second, $\mathrm{CaCCs}$ might also fulfill a role in chloride movements as has been proposed for some epithelial cells whose activation would lead to transepithelial transport of salt together with water. Well-characterized examples, particularly of CaCCs of epithelial tissues, are summarized below, but comprehensive reviews can be consulted for more instances of the proposed functions of CaCCs (4-6). Whenever possible, reviews rather than original contributions are cited due to space constraints.

CaCCs have been proposed to be important in a variety of functions in different cells or tissues. For instance, in olfactory neurons CaCCs are believed to amplify the odorant-induced signal by virtue of their activation by an elevation in intracellular calcium (7). Less well characterized, but nevertheless important roles are played by CaCCs in the adaptation of taste receptors and in the modulation of

Correspondence: M.I. Niemeyer, Centro de Estudios Científicos (CECS), Avenida Arturo Prat, 514, Valdivia, Chile.

E-mail: miniemeyer@cecs.cl

Research supported by Fondecyt (\#1061069 and \#3085023). The Centro de Estudios Científicos (CECS) is funded by the Chilean Government through the Millennium Science Initiative and the Centers of Excellence Base Financing Program of Conicyt. CECS is also supported by a group of private companies, which at present includes Arauco, Empresas CMPC and Telefónica del Sur. CIN is funded by Conicyt and the Gobierno Regional de Los Ríos. 
photoreceptors $(4,5)$. The function of CaCCs in other neurons remains less well explored. CaCCs are also known to play important roles in myocytes from cardiac and smooth muscle. In cardiac myocytes, since the equilibrium potential for $\mathrm{Cl}^{-}$is around $-50 \mathrm{mV}$, activation of CaCCs has been suggested to contribute to delayed afterdepolarization (8). In smooth muscle cells, CaCCs are thought to take part in the regulation of the myogenic tone through membrane depolarization and in neurotransmitter- and local mediatorregulated contractility (9).

In oocytes, CaCCs play a role in the prevention of polyspermy in amphibians, i.e., the blockade of sperm penetration once an initial fertilization has occurred. It is known that sperm penetration is accompanied by a rapid increase in intracellular calcium that leads to a prolonged and profound membrane depolarization. This membrane depolarization has been attributed to a sustained activation of CaCCs which, because intracellular chloride is above equilibrium, shifts the membrane potential to a more positive value (4).

CaCCs have been proposed to be involved in transepithelial transport, particularly in chloride and fluid secretion elicited by calcium-mobilizing agonists. The function of these $\mathrm{CaCCs}$ has been very well characterized in the fluid and electrolyte secretory processes of glandular tissues such as the salivary gland and the pancreas but it is less well clearly defined in the respiratory epithelium and remains controversial in the intestinal epithelium.

The characterization of $\mathrm{CaCC}$ s has relied mainly upon macroscopic current recordings since many reports indicate that their single channel conductance is too small for easy recording and maneuvers such as patch excision often lead to channel rundown (5).

\section{CaCCs of secretory glands}

The main electrophysiological characteristics of what has been called classical CaCCs (4) were already identified in lacrimal glands by Marty et al. in 1984 (10) and their kinetic and other properties were reported in some detail in 1986 (11). Lacrimal gland CaCCs were activated by intracellular calcium in the range of 0.5 to $2 \mu \mathrm{M}$ with a steep dose-response curve suggestive of cooperativity. The channels were markedly outward rectifying and activated by depolarization at low intracellular calcium. At the higher $2-\mu \mathrm{M}$ intracellular calcium, the channels became virtually voltage-insensitive and non-rectifying. The single channel conductance of lacrimal gland CaCCs was estimated to be 1-2 pS by noise analysis. Lacrimal gland CaCCs could be activated by carbachol, presumably by physiologically meaningful intracellular calcium mobilization, and had a selectivity sequence, derived from shifts in reversal potential, of $\mathrm{I}^{-}>\mathrm{NO}_{3}^{-}>\mathrm{Br}^{-}>\mathrm{Cl}^{-}>\mathrm{F}^{-}>$isethionate, methanesulfonate $>$ glutamate. Arreola et al. in 1996 (12) reported similar currents in salivary parotid acinar cells and proposed them as the mediators of chloride efflux activated by increased intracellular calcium during fluid secretion. More recently, the channels of Xenopus oocytes have been revisited using excised insideout patches of membrane obtained from regions known to have a high density of channels (13). This approach allowed measurements of macroscopic chloride currents, affording at the same time easy manipulation of intracellular calcium levels in defined solutions. The conclusion from this work is that the characteristics of oocyte CaCCs are very similar to those of lacrimal or parotid acinar cells. They provide, however, confirmation that these channels are directly activated by intracellular calcium without any need for invoking a calcium-dependent phosphorylation step. A comparison between $\mathrm{CaCC}$ activated in salivary gland acinar cells and human colonic carcinoma cells highlighted the lack of need for the mediation by calmodulin-dependent protein kinase (CaM kinase) II for activation in the native cells compared with the transformed colonic epithelium (14).

\section{Airway epithelial CaCCs}

Using human nasal epithelium mounted in Ussing chambers, it has been shown that stimulation of $\mathrm{P} 2 \mathrm{Y}_{2}$ receptors by ATP or uridine-5'-triphosphate (UTP) added to the apical side induces a secretory transepithelial current that has been attributed to the opening of apical CaCCs exclusively, as it is also observed using nasal tissue samples from human patients affected by cystic fibrosis (15). Interestingly, a serosal purinergic stimulus also induced anion secretion, but this corresponds to the opening of cystic fibrosis transmembrane conductance regulator (CFTR) channels as it is totally absent from cystic fibrosis tissue. Thus, in this epithelium it is possible to activate different anion conductances with the same agonist depending on the side of the membrane where the stimulus is applied. The presence of CaCCs does not seem to be sufficient to alleviate the effects of cystic fibrosis in humans, but mice deficient in CFTR show no marked respiratory pathology and it has been speculated that a more predominant calcium-dependent chloride secretion in murine airways might compensate for the lack of CFTR in the knockout mouse. As recently discussed by Kunzelmann et al. (16), functional microdomains formed through the interaction of calcium-mobilizing receptors, store-operated calcium channels and $\mathrm{CaCC}$ might account for the observations of sidedness of agonist effects in respiratory, and perhaps other, epithelia. Recent data show that proinflammatory stimuli may significantly modify ion transport in the airway epithelium and therefore the properties of the airway surface fluid.

Further evidence for the presence of a separate pathway for calcium-dependent chloride secretion in the respiratory epithelium comes from studies of the effect of IL-4, a cytokine related to the pathogenesis of asthma, in human bronchial cells in vitro (17). Long-term treatment of the cells with IL-4 causes a 2-fold increase in cAMP-dependent, CFTR-mediated secretion, but enhances 4- to 5-fold calcium-dependent chloride secretion measured as that current activated by api- 
cal UTP. The enhancement of UTP-elicited chloride current was also present in cells from cystic fibrosis patients, ruling out a participation of CFTR.

\section{Intestinal epithelium CaCCs}

Studies of transepithelial secretion of fluid and electrolytes in the intestine have historically led the way to our understanding of basic concepts of this important physiological process and its associated pathophysiology (18). It is well known that CAMP-mediated agonists, and Vibrio cholera toxin, are able to elicit CFTR-mediated chloride secretion. Calcium-mobilizing agonists, including cholinergic muscarinic agonists, nucleotides and histamine, are also capable of eliciting intestinal chloride secretion.

The idea of the intestinal epithelium as a site of robust expression of $\mathrm{CaCC}$ s came from experiments on epithelial cell lines of colonic carcinoma origin. For instance, $\mathrm{T}_{84}$ cells were observed to possess currents of the CaCC type that were in addition dependent upon CaM kinase II $(19,20)$. The presence of $\mathrm{CaCC}$ in native intestinal epithelium has been more difficult to demonstrate and there is a body of evidence suggesting that, although calcium-activated intestinal chloride requires basolateral calcium-dependent potassium channels, it is absolutely dependent upon the activity of apical membrane CFTR chloride channels (21). There are some reasons, however, to consider a degree of possible participation of $\mathrm{CaCCs}$ in intestinal chloride secretion.

The first is that in CFTR knockout mice, which usually die of intestinal obstruction, the severity of the disease is genetically determined, since different inbred mouse strains obtained from backcrosses and intercrosses of Cftr-deficient mice show different survival rates (22). Genetic analysis has demonstrated that there is a modifier locus for cystic fibrosis severity (cystic fibrosis modulator locus 1, Cfm1) near the centromere of chromosome 7. Comparative maps of the locus found on mouse chromosome 7 with other mammals shows syntenic regions in rat and human chromosomes. The human syntenic region is located within chromosome 19 , in a locus that has been related to meconium ileus, an intestinal obstruction observed in nearly $20 \%$ of cystic fibrosis patients during childhood (23). It is conceivable that the severity of the intestinal disease could be determined by the genetic background and that the relevant trait might be the differential expression of CaCCs or other chloride channels (24). Secondly, the presence of $\mathrm{CaCCs}$ has been repeatedly reported in the rat intestinal epithelium $(25,26)$ and CaCCs have been suggested to act as mediators of rotavirus-induced diarrhea in 7- to 14-day-old mouse pups (27).

We have re-examined the issue of the possible presence of CaCCs as mediators of calcium-dependent chloride secretion in mouse colon. The best known blocker of CaCCs known to date is niflumic acid, which effectively blocks Xenopus oocyte CaCCs in the $10-\mu \mathrm{M}$ range (4). Figure $1 \mathrm{~A}$ shows a recording of the transepithelial potential difference $\left(V_{\text {te }}\right)$ of mouse colon. The addition of basolateral carbachol reveals a robust chloride secretion response seen as a

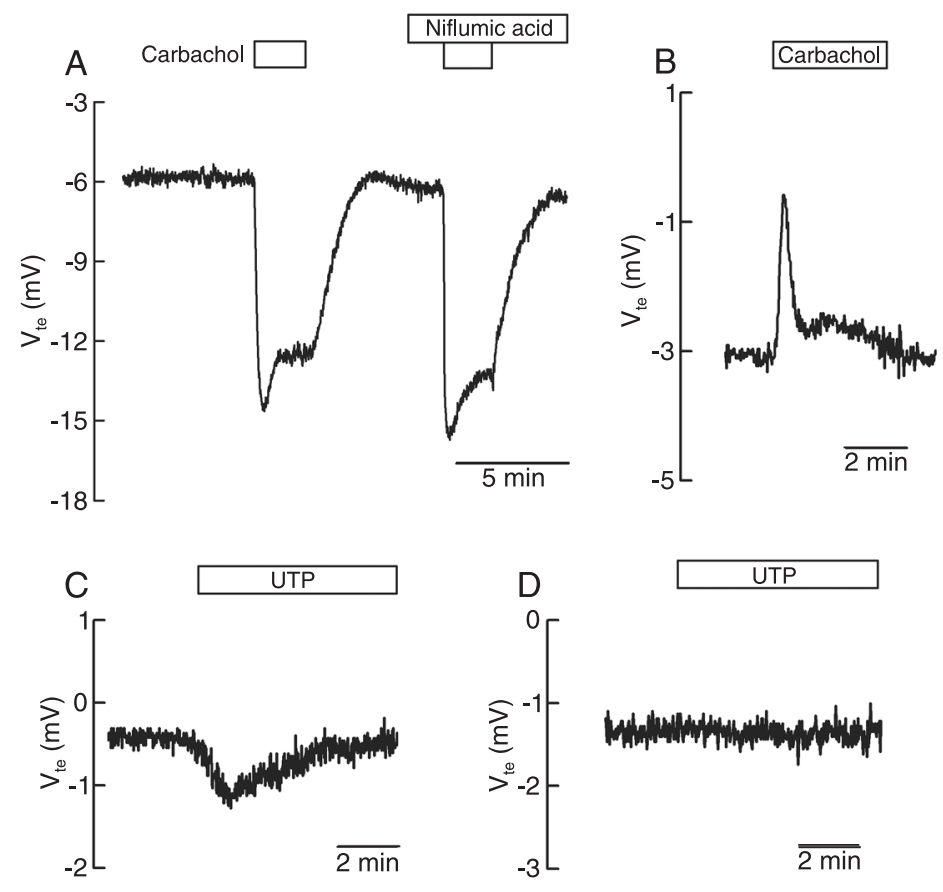

Figure 1. Calcium-activated chloride secretion in mouse colon. The traces show continuous recordings of transepithelial potential difference $\left(\mathrm{V}_{\mathrm{te}}\right)$ taken from stripped tissues mounted in Ussing chambers under current-clamp conditions. A change in $V_{\text {te }}$ in the negative direction corresponds to anion secretion, while positive shifts in $\mathrm{V}_{\text {te }}$ are consistent with cation (generally potassium) secretion (21). A, Addition of basolateral carbachol $(100 \mu \mathrm{M})$ to the colon of a wild-type mouse reveals a robust chloride secretion response. The effect was not inhibited by the calcium-activated chloride channel blocker niflumic acid added to the apical side of the tissue at a concentration of 150 $\mu \mathrm{M}$. When colon epithelium from a mouse deficient in cystic fibrosis transmembrane conductance regulator (CFTR) (58) was similarly challenged with carbachol there was no response that could be linked to chloride secretion. Instead, a positive spike that corresponds to potassium secretion $(21,28)$ is observed. In both experiments the tissues were previously incubated with tetrodotoxin (TTX) to inhibit stimulation of chloride secretion via residual enteric neurons, with amiloride to inhibit sodium absorptive currents via $\mathrm{ENaC}$, with forskolin to increase cAMP levels and activate CFTR, and with chromanol 293B to inhibit CAMP-activated potassium channels, thus preventing any cAMP-activated chloride secretion (21). $C$ and $D$ show the effect of basolateral uridine-5'-triphosphate (UTP) at $100 \mu \mathrm{M}$ on $\mathrm{V}_{\text {te }}$ of colonic epithelium from wild-type (C) and CFTR(-/-) (D) mice. The epithelia used in experiments $C$ and $D$ were previously incubated with TTX and amiloride only. The traces illustrated are representative experiments. Each experiment was repeated at least three times using tissues from different animals, with similar results. 
negative deflection in $V_{\text {te }}$. Niflumic acid was unable to affect any portion of the calcium-activated chloride secretion elicited with the muscarinic agonist. Figure 1B shows carbachol stimulation in a colonic epithelium from a CFTR knockout mouse. There was no evidence for anion secretion, but positive displacements in $\mathrm{V}_{\text {te }}$ suggested that in the absence of CFTR a potassium secretory process had been unmasked.

It has been demonstrated that the addition of ATP or UTP to the apical side of the mouse colonic epithelial layers induces a rapid and transient increase in potassium secretion by activation of maxi-potassium channels (28). The receptors mediating this effect are $P 2 Y_{2}$ and $P 2 Y_{4}$ receptors, as demonstrated by the use of tissues from the respective knockout mice (29). The stimulation of colonic epithelium by the basolateral addition of UTP induced a small electrical response that could be associated with calcium-dependent chloride secretion in the tissue from mice of CD1/SV129 hybrid background (29). We have recorded similar responses in our experiments with colonic epithelium from BALBc/Black Swiss animals (see Figure 1C). However, this small anion secretion response was absent in the colon of CFTR knockout mice (Figure 1D), suggesting that the observed change in $V_{\text {te }}$ was due to the activation of the cAMP-dependent CFTR chloride channel. It is interesting to note that when the potassium current activated by apical UTP is abolished by knocking out either the P2Y receptors or the maxi-potassium channels, or by inhibiting with apical barium $(28,29)$, the small negative change of $V_{\text {te }}$ is still present. This suggests that differential activation of CFTR and the $\mathrm{CaCC}$ s seen in the airway epithelium (see above) is not taking place in the colon.

We have also studied carbachol-induced chloride secretion in colonic tissue from 8- to 14-day-old CFTR(-/-) pups to test for the presence of calcium-dependent chloride secretion as suggested by work on rotavirus-induced diarrhea (27). In no instance did we detect an electrophysiological response consistent with calcium-dependent chloride secretion.

These new observations reinforce the view that if $\mathrm{CaCCs}$ are present in the intestine, they are not involved in chloride secretion in any major way, and that any form of anion secretion in the intestine requires the activity of CFTR chloride channels.

\section{Possible molecular counterparts of calcium-activated chloride channels}

Further advance in our understanding of the physiology of $\mathrm{CaCC}$ s has been hampered by the lack of reliably identified molecular counterparts of these channels. The potential advantages of such an identification include the possibility to study their physiological and pathophysiological roles by knocking them out specifically, detailed structure function studies and the potential identification of genuinely specific inhibitors. At least three candidate proteins have been proposed as the molecular counterparts of $\mathrm{CaCCs}$, as described below.

The CLCA family of proteins, for which there are examples of cloned putative $\mathrm{CaCC}$ channels from respiratory and intestinal tissues $(30,31)$. There is, however, great skepticism over the function of CLCAs as chloride channels $(32,33)$, among other things because some of them do not appear to be integral membrane proteins but seem to be soluble, secreted proteins.

The CIC-3 member of the CIC family of chloride channels and transporters has been proposed to underlie the CAMKII-activated chloride current of the type seen in T84 and other colonic carcinoma cells $(34,35)$. The properties of the currents reported for $\mathrm{ClC}-3$, however, differ from those typically described for $\mathrm{CaCCs}$ and a normal $\mathrm{CaCC}$ activity has been shown in parotid acinar cells from a $\mathrm{CIC}-3$ knockout mouse (36).

Bestrophin, a protein product of the vitelliform macular disease VMD gene, was shown to form oligomeric chloride channels sensitive to intracellular calcium. In addition, each of 15 disease-associated mutants of VMD was shown to exhibit reduced or null membrane current. Four of these mutant bestrophins had a dominant negative effect on wildtype membrane current, consistent with the nature of the disease (37). The position of bestrophins as genuine chloride channels has received strong support from a variety of experimental approaches, probably the strongest being sitedirected mutagenesis coupled with functional experiments on Best2 demonstrating the molecular basis of permeation and blockade properties of multiatomic anion thiocyanate (38). Although fulfilling the essential requirement of being chloride channels, bestrophins have fallen out of favor as candidate molecules to underlie the classical CaCCs as they do not share some of their functional characteristics. As reviewed by Hartzell et al. (39), bestrophins lack the characteristic outwardly rectifying kinetics of CaCCs, Best1 and Best2 have intracellular calcium affinities 10-fold higher than those of classical CaCCs, have not been shown to be activated by calcium-mobilizing agonists and, unlike CaCCs, are also activated by increases in cell volume, which might place them in the category of volume-regulated anion channels (40).

\section{TMEM16 family of membrane proteins as CaCCs}

Last year saw a flurry of activity in the field of the CaCCs with the almost simultaneous publication of three papers reporting that TMEM16A is a bona fide CaCC (4143). These publications have elicited much interest in the membrane biology field as it turns out that the functional expression of TMEM16A in heterologous systems yielded a conductance that for the first time showed the "classical" characteristics of the CaCCs. 
Upon discovery of the anion channel nature of TMEM16A, Yang et al. (41) changed the name of TMEM16 to Anoctamin (abbreviated ANO), incorporating the concept that they might be anion channels and the fact that they have eight putative transmembrane segments. We have adhered to the TMEM16 nomenclature here because the eight transmembrane domain concepts might be transient, as illustrated by the drastic change in number of $\alpha$-helices upon publication of the first X-ray structure of a CIC transport protein (44) and the potentially embarrassing meaning of the abbreviation in Spanish.

Yang et al. (41) took the apparently straightforward approach of mining the public domain databases for genes coding for proteins with more than two predicted transmembrane domains and considered to be putative transporters or channels. They fell upon TMEM16A, which had 8 predicted transmembrane domains and 10 homologues in man. TMEM16A co-expressed with endothelin receptor A endowed HEK293 cells with robust endothelin-induced chloride currents that were outwardly rectified at low activation levels but non-rectifying at maximal activation. The currents had the typical selectivity of $\mathrm{CaCCs}$ and could be blocked by chloride channel blockers, among them niflumic acid. That TMEM16A is a genuine chloride channel can be deduced by site-directed mutagenesis of a region identified as a possible pore loop. Reversing the charge of three highly conserved basic residues in this region changed TMEM16A from anion- to cation-selective and blocking a trio of cysteine residues inhibited the current. When studied in inside-out patches, intracellular calcium activated the channel in a cooperative manner much as found before for so-called classical CaCCs. TMEM16A was found to be present in cells and tissues associated with $\mathrm{CaCC}$ expression. These included epithelial cells of pulmonary bronchioles, pancreatic acinar cells, kidney proximal tubules, all retinal cell layers, most sensory neurons, Leydig cells of testis, and the apical membranes of submandibular gland acinar cells. Using an siRNA approach Yang at al. (41) were also able to decrease the expression of TMEM16A in submandibular glands of mice and to reduce pilocarpine-induced saliva output.

Caputo et al. (42) took advantage of their previous observation that long-term stimulation with cytokines such as IL-4 and IL-13 of cell cultures from epithelial bronchial human cells results in the enhancement of calcium-dependent chloride secretion activated by UTP (see above) to search for possible enhanced transcribed genes in microarray analyses. Among other gene products, they identified TMEM16A, which was enhanced 7-fold by treatment with IL-4. They then used an siRNA approach to show that UTP-evoked CaCC activity enhanced by IL-4 in cystic fibrosis cells from pancreas and bronchial epithelium was markedly reduced in cells transfected with siRNA against TMEM16A. siRNA against the TMEM16 family members TMEM16F or TMEM16K had no effect. Transfection of
TMEM16A cDNA into Fisher rat thyroid or HEK293 cells produced membrane protein expression and niflumic acidsensitive chloride currents with typical CaCC-like outwardly rectifying characteristics. Caputo et al. (42) also validated the genuineness of TMEM16A as a chloride channel by sitedirected mutagenesis, but targeted putative transmembrane domains rather than the pore domain identified by Yang et al. (41). Neutralization of basic amino acids in transmembrane domains 5 and 8 led to marked current reduction, while neutralizing R563 in transmembrane domain 3 led to altered kinetics. Mutation Q757A in transmembrane domain 6 abolished voltage dependence and altered the selectivity of channels associated to TMEM16A expression.

Schroeder et al. (43) exploited the fact that oocytes from the axolotl Ambystoma mexicanum lack CaCCs (and can be therefore polyspermic!) to use them as an expression cloning system for Xenopus laevis oocyte CaCC mRNA. This strategy led them to discover xTMEM16A, the expression of which generated calcium-activated chloride currents with the typical marked voltage dependence and outward rectification of Xenopus oocyte CaCC. As expected, the currents became less dependent upon voltage and showed little rectification at high intracellular calcium levels. Calcium dependence had the expected cooperative behavior, which became steeper the more positive the potential. Currents could also be activated by carbachol-induced intracellular calcium mobilization. The current was most strongly inhibited by niflumic acid and also by 4,4'-diisothiocyanatostilbene2,2'-disulfonic acid (DIDS) with a dissociation constant of 29 $\mu \mathrm{M}$. Schroeder et al. (43) also identified the mouse isoform of TMEM16A and, in addition to showing its functional expression in HEK293 cells, they demonstrated their presence in mammary and salivary glands by in situ hybridization.

Almost coincidental with these reports, and apparently unaware of the identification of TMEM16A as a CaCC, Rock et al. (45) had reported a TMEM16A knockout mouse. All knockout homozygous mice died within one month of birth exhibiting severe tracheomalacia with gaps in the tracheal cartilage rings. Rock et al. (45) proposed that the cartilage ring defect observed in TMEM16A knockout animals was secondary to an improper stratification of the embryonic tracheal epithelium or the abnormal trachealis muscle. It is tempting to speculate that the absence of calcium-mediated chloride secretion in the trachea of mutant animals is the cause of the ensuing malformation.

\section{Are all TMEM16 proteins chloride channels?}

The identification of TMEM16A as a $\mathrm{CaCC}$ with all the long-sought characteristics of this important ion channel opens the way for its structure-function study by site-directed mutagenesis and functional assay, the resolution of its structure if crystallization of a TMEM16 could be achieved and further investigation of its knockout in mice (45) to probe its role in tissues where it is prominently expressed. 
Particularly, this last approach will help elucidate the importance of the other members of the TMEM16 family in the physiology of diverse organs.

Another important question is whether other TMEM16s also function as chloride channels. In the mouse, for example, there are at least eleven members of the TMEM16 family (see dendrogram in Figure 2), with TMEM16B being the most closely related to the one identified as $\mathrm{CaCC}$. Also, the question remains as to their oligomeric structure. It is possible that more than one isoform might coexist at the

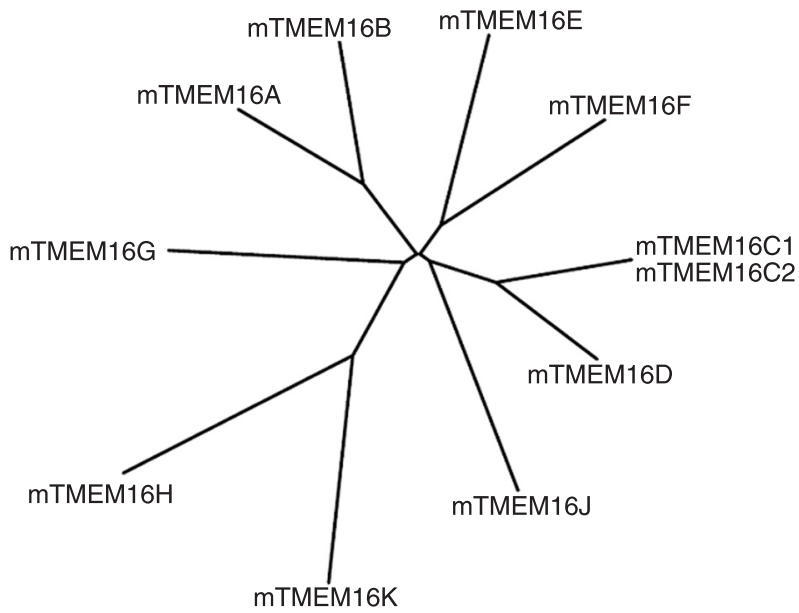

Figure 2. Phylogenetic tree of the mouse TMEM16 family members. Mouse sequences were obtained from the GeneBank and had the following accession numbers: BC062959.1 (TMEM16A), BC033409.1 (TMEM16B), NM_178773.4 (TMEM16D), NM_177694.5 (TMEM16E), NM001081556.1 (TMEM16C, transcript variant 1), NM_001128103.1 (TMEM16C, transcript variant 2), NM_175344.3 (TMEM16F), NM 207031.1 (TMEM16G), BC027790.1 (TMEM16H), NM 178381.3 (TMEM16J), NM_133979 (TMEM16K). The sequences were analyzed using the phylogeny.fr software (http://www.phylogeny.fr/version2_cgi/ index.cgi). same cell location. There is a clear need to couple these possible research avenues with an examination of which cells and tissues express which TMEM16 isoforms to obtain insight about novel functions for these proteins. This type of question needs to be addressed also in tissues that do not appear to harbor classical $\mathrm{CaCC}$, such as the intestine, as this might lead to fresh views about the potential functions of this interesting family of proteins. As a first step in tackling this question we have examined the presence of TMEM16 transcripts of all eleven members present in colonic epithelium. Specific primers were designed and the results of PCR amplification are shown in Figure 3. Seven of the eleven identified TMEM16 transcripts, namely those of TMEMs $\mathrm{A}, \mathrm{B}, \mathrm{E}, \mathrm{F}, \mathrm{G}, \mathrm{J}$, and $\mathrm{K}$, are present in colonic epithelium. The presence of TMEM16A is puzzling in view of the lack of calcium-activated chloride secretion in intestinal tissue discussed above. Although there is no evidence for $\mathrm{CaCCs}$ mediating calcium-activated intestinal secretion (46), the presence of a carbachol-activated chloride conductance in crypts from CFTR knockout mice has been deduced from agonist-elicited changes in cell volume (47). In view of the absence of calcium-dependent chloride secretion in this epithelium, it is possible that what was observed corresponds to the activation of basolateral membrane chloride channels, therefore having an impact on cell volume through $\mathrm{KCl}$ efflux but not on transepithelial secretion. Chloride channels in such a location have been reported in both small and large intestine (48-50). Future work will be needed to determine whether TMEM16A, or indeed the other TMEM16 congeners present in colon epithelium, make a contribution to the chloride conductance of these cells.

\section{Final remarks}

The identification of TMEM16A protein as a $\mathrm{CaCC}$ with all the expected characteristics of the calcium-activated chloride channels expressed in various epithelial, neuronal

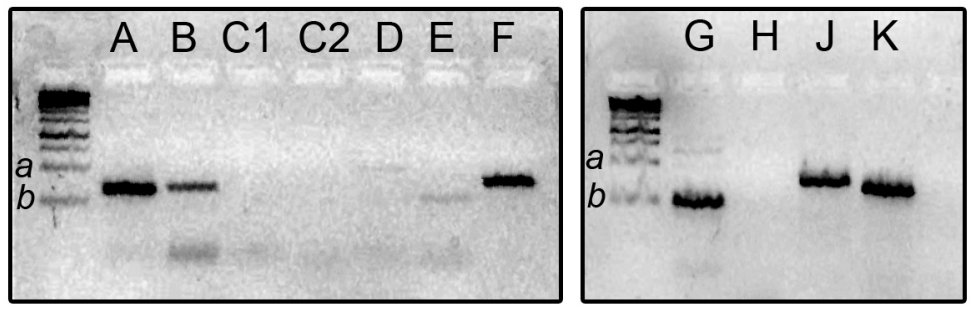

Figure 3. PCR detection of mRNAs for TMEM16 family members in colonic tissue. Colonic epithelium was stripped of its muscle layers and mRNA was isolated and reverse transcribed (59). PCR was performed using specific primers for members of the TMEM16 family using a single cDNA sample. Lanes contained a 1-kB ladder ( $a$ and $b$ correspond to the 500- and 250-bp marker, respectively) and then TMEM16A (324 bp), TMEM16B (330 bp), TMEM16C1 (341 bp), TMEM16C2 (320 bp), TMEM16D (493 bp), TMEM16E (226 bp), TMEM16F (380 bp), 1-kB ladder, TMEM16G (248 bp), TMEM16H (296 bp), TMEM16J (374 bp), and TMEM16K (329 bp). The PCR conditions included 35 cycles with annealing temperature of $55^{\circ} \mathrm{C}$. A representative experiment of three different ones performed with independent cDNA samples is shown. Control experiments were carried out using whole brain, and olfactory epithelium obtained from mice and rats confirmed the ability of all primer sets to amplify the expected products (González C and Bacigalupo J, unpublished data). 
and other cells opens the way for the detailed molecular study of a whole new family of potential chloride channels. It has been pointed out (43) that some TMEM16 family members could fulfill tasks in intracellular membranes. An example of this is Drosophila Aberrant $X$ segregation protein that is present in membranes surrounding the meiotic spindle (51).

The level of CaCC activity in epithelia of CFTR knockout mice correlates with the severity of disease $(46,52)$. CaCC-mediated secretion is not affected by cystic fibrosis in humans $(53,54)$. Modulating $\mathrm{CaCC}$ activity, for example in the airways, could help alleviate some of the pulmonary effects of the disease, a course of action that might be facilitated by the identification of TMEM16A as the molecular counterpart of those channels.

There is also evidence that TMEM16A gene is present in various cancers including gastrointestinal stromal tumors (55-57). It is of course too early to say whether the channel function of TMEM16A is related to tumorigenesis and

\section{References}

1. Miledi R. A calcium-dependent transient outward current in Xenopus laevis oocytes. Proc R Soc Lond B Biol Sci 1982; 215: 491-497.

2. Barish ME. A transient calcium-dependent chloride current in the immature Xenopus oocyte. J Physiol 1983; 342: 309325.

3. Bader CR, Bertrand D, Schwartz EA. Voltage-activated and calcium-activated currents studied in solitary rod inner segments from the salamander retina. J Physiol 1982; 331: 253-284.

4. Hartzell C, Putzier I, Arreola J. Calcium-activated chloride channels. Annu Rev Physiol 2005; 67: 719-758.

5. Frings S, Reuter D, Kleene SJ. Neuronal $\mathrm{Ca}^{2+}$-activated $\mathrm{Cl}^{-}$ channels - homing in on an elusive channel species. Prog Neurobiol 2000; 60: 247-289.

6. Melvin JE, Yule D, Shuttleworth T, Begenisich T. Regulation of fluid and electrolyte secretion in salivary gland acinar cells. Annu Rev Physiol 2005; 67: 445-469.

7. Reisert J, Bauer PJ, Yau KW, Frings S. The Ca-activated Cl channel and its control in rat olfactory receptor neurons. $J$ Gen Physiol 2003; 122: 349-363.

8. Zygmunt AC, Goodrow RJ, Weigel CM. INaCa and ICl(Ca) contribute to isoproterenol-induced delayed after depolarizations in midmyocardial cells. Am J Physiol 1998; 275: H1979-H1992.

9. Large WA, Wang Q. Characteristics and physiological role of the $\mathrm{Ca}^{2+}$-activated $\mathrm{Cl}^{-}$conductance in smooth muscle. $\mathrm{Am}$ J Physiol 1996; 271: C435-C454.

10. Marty A, Tan YP, Trautmann A. Three types of calciumdependent channel in rat lacrimal glands. J Physiol 1984; 357: 293-325.

11. Evans MG, Marty A. Calcium-dependent chloride currents in isolated cells from rat lacrimal glands. J Physiol 1986; 378 : 437-460.

12. Arreola J, Melvin JE, Begenisich T. Activation of calciumdependent chloride channels in rat parotid acinar cells. $J$ whether channel inhibitors or activators might be of any potential therapeutic use.

Independently of the possible future directions of the research on TMEM16 proteins, their discovery as the chloride channel molecules behind $\mathrm{CaCC}$ activity represents a great triumph of scientific perseverance and ingenuity. The varied approaches used by the three independent research groups also augur well for the solidity of the discovery, a quality that has not often been associated with the difficult quest for molecules behind the activities in the field of anion channels.

\section{Acknowledgments}

We are grateful to Carolina González for allowing us to quote her TMEM16 PCR results and to David Jaime of the CECS Mouse Genomic Facility for his untiring help with the animals.

13. Kuruma A, Hartzell $\mathrm{HC}$. Bimodal control of a $\mathrm{Ca}^{2+}$-activated $\mathrm{Cl}^{-}$channel by different $\mathrm{Ca}^{2+}$ signals. J Gen Physiol 2000; 115: 59-80.

14. Arreola J, Melvin JE, Begenisich T. Differences in regulation of $\mathrm{Ca}^{2+}$-activated $\mathrm{Cl}^{-}$channels in colonic and parotid secretory cells. Am J Physiol 1998; 274: C161-C166.

15. Paradiso AM, Ribeiro CM, Boucher RC. Polarized signaling via purinoceptors in normal and cystic fibrosis airway epithelia. J Gen Physiol 2001; 117: 53-67.

16. Kunzelmann K, Milenkovic VM, Spitzner M, Soria RB, Schreiber R. Calcium-dependent chloride conductance in epithelia: is there a contribution by Bestrophin? Pflügers Arch 2007; 454: 879-889.

17. Galietta LJ, Pagesy P, Folli C, Caci E, Romio L, Costes B, et al. IL-4 is a potent modulator of ion transport in the human bronchial epithelium in vitro. J Immunol 2002; 168: 839845.

18. Field M. Intestinal electrolyte secretion. History of a paradigm. Arch Surg 1993; 128: 273-278.

19. Cliff WH, Frizzell RA. Separate $\mathrm{Cl}^{-}$conductances activated by cAMP and $\mathrm{Ca}^{2+}$ in $\mathrm{Cl}^{-}$-secreting epithelial cells. Proc Natl Acad Sci U S A 1990; 87: 4956-4960.

20. Worrell RT, Frizzell RA. CaMKII mediates stimulation of chloride conductance by calcium in T84 cells. Am J Physiol 1991; 260: C877-C882.

21. Flores CA, Melvin JE, Figueroa CD, Sepúlveda FV. Abolition of $\mathrm{Ca}^{2+}$-mediated intestinal anion secretion and increased stool dehydration in mice lacking the intermediate conductance $\mathrm{Ca}^{2+}$-dependent $\mathrm{K}^{+}$channel Kcnn4. J Physiol 2007; 583: 705-717.

22. Zielenski J, Corey M, Rozmahel R, Markiewicz D, Aznarez I, Casals $T$, et al. Detection of a cystic fibrosis modifier locus for meconium ileus on human chromosome 19q13. Nat Genet 1999; 22: 128-129.

23. Rozmahel R, Wilschanski M, Matin A, Plyte S, Oliver M, 
Auerbach W, et al. Modulation of disease severity in cystic fibrosis transmembrane conductance regulator deficient mice by a secondary genetic factor. Nat Genet 1996; 12: 280-287.

24. Gyomorey K, Garami E, Galley K, Rommens JM, Bear CE. Non-CFTR chloride channels likely contribute to secretion in the murine small intestine. Pflügers Arch 2001; 443 (Suppl 1): S103-S106.

25. Schultheiss G, Siefjediers A, Diener M. Muscarinic receptor stimulation activates a $\mathrm{Ca}^{2+}$-dependent $\mathrm{Cl}^{-}$conductance in rat distal colon. J Membr Biol 2005; 204: 117-127.

26. Hennig B, Schultheiss G, Kunzelmann K, Diener M. Ca ${ }^{2+}-$ induced $\mathrm{Cl}^{-}$efflux at rat distal colonic epithelium. J Membr Biol 2008; 221: 61-72.

27. Morris AP, Scott JK, Ball JM, Zeng CQ, O'Neal WK, Estes MK. NSP4 elicits age-dependent diarrhea and $\mathrm{Ca}^{2+}$ mediated I- influx into intestinal crypts of CF mice. Am J Physiol 1999; 277: G431-G444.

28. Sausbier M, Matos JE, Sausbier U, Beranek G, Arntz C, Neuhuber W, et al. Distal colonic $\mathrm{K}^{+}$secretion occurs via BK channels. J Am Soc Nephrol 2006; 17: 1275-1282.

29. Matos JE, Robaye B, Boeynaems JM, Beauwens R, Leipziger $\mathrm{J} \mathrm{K}^{+}$secretion activated by luminal P2Y2 and P2Y4 receptors in mouse colon. J Physiol 2005; 564: 269279.

30. Cunningham SA, Awayda MS, Bubien JK, Ismailov II, Arrate MP, Berdiev BK, et al. Cloning of an epithelial chloride channel from bovine trachea. J Biol Chem 1995; 270: 3101631026.

31. Gaspar KJ, Racette KJ, Gordon JR, Loewen ME, Forsyth GW. Cloning a chloride conductance mediator from the apical membrane of porcine ileal enterocytes. Physiol Genomics 2000; 3: 101-111.

32. Jentsch TJ, Stein V, Weinreich F, Zdebik AA. Molecular structure and physiological function of chloride channels. Physiol Rev 2002; 82: 503-568.

33. Eggermont J. Calcium-activated chloride channels: (un) known, (un)loved? Proc Am Thorac Soc 2004; 1: 22-27.

34. Huang P, Liu J, Di A, Robinson NC, Musch MW, Kaetzel MA, et al. Regulation of human CLC-3 channels by multifunctional $\mathrm{Ca}^{2+} /$ calmodulin-dependent protein kinase. J Biol Chem 2001; 276: 20093-20100.

35. Robinson NC, Huang P, Kaetzel MA, Lamb FS, Nelson DJ. Identification of an N-terminal amino acid of the CLC-3 chloride channel critical in phosphorylation-dependent activation of a CaMKII-activated chloride current. J Physiol 2004; 556: 353-368.

36. Arreola J, Begenisich T, Nehrke K, Nguyen HV, Park K, Richardson $\mathrm{L}$, et al. Secretion and cell volume regulation by salivary acinar cells from mice lacking expression of the Clcn3 $\mathrm{Cl}^{-}$channel gene. J Physiol 2002; 545: 207-216.

37. Sun H, Tsunenari T, Yau KW, Nathans J. The vitelliform macular dystrophy protein defines a new family of chloride channels. Proc Natl Acad Sci U S A 2002; 99: 4008-4013.

38. Qu Z, Fischmeister R, Hartzell C. Mouse bestrophin-2 is a bona fide $\mathrm{Cl}^{-}$channel: identification of a residue important in anion binding and conduction. J Gen Physiol 2004; 123: 327-340.

39. Hartzell HC, Qu Z, Yu K, Xiao Q, Chien LT. Molecular physiology of bestrophins: multifunctional membrane proteins linked to Best disease and other retinopathies. Physiol Rev 2008; 88: 639-672.
40. Valverde MA, Mintenig GM, Sepúlveda FV. Differential effects of tamoxifen and $\mathrm{I}$ - on three distinguishable chloride currents in T84 intestinal cells. Pflügers Arch 1993; 425: 552-554.

41. Yang YD, Cho H, Koo JY, Tak MH, Cho Y, Shim WS, et al. TMEM16A confers receptor-activated calcium-dependent chloride conductance. Nature 2008; 455: 1210-1215.

42. Caputo A, Caci E, Ferrera L, Pedemonte N, Barsanti C, Sondo E, et al. TMEM16A, a membrane protein associated with calcium-dependent chloride channel activity. Science 2008; 322: 590-594.

43. Schroeder BC, Cheng T, Jan YN, Jan LY. Expression cloning of TMEM16A as a calcium-activated chloride channel subunit. Cell 2008; 134: 1019-1029.

44. Dutzler R, Campbell EB, Cadene M, Chait BT, MacKinnon $\mathrm{R}$. X-ray structure of a $\mathrm{CIC}$ chloride channel at 3.0 A reveals the molecular basis of anion selectivity. Nature 2002; 415: 287-294.

45. Rock JR, Futtner CR, Harfe BD. The transmembrane protein TMEM16A is required for normal development of the murine trachea. Dev Biol 2008; 321: 141-149.

46. Clarke LL, Grubb BR, Yankaskas JR, Cotton CU, McKenzie A, Boucher RC. Relationship of a non-cystic fibrosis transmembrane conductance regulator-mediated chloride conductance to organ-level disease in $\mathrm{Cftr}(-/-)$ mice. Proc Natl Acad Sci U S A 1994; 91: 479-483.

47. Valverde MA, O'Brien JA, Sepúlveda FV, Ratcliff R, Evans $\mathrm{MJ}$, Colledge WH. Inactivation of the murine cftr gene abolishes cAMP-mediated but not $\mathrm{Ca}^{2+}$-mediated secretagogueinduced volume decrease in small-intestinal crypts. Pflügers Arch 1993; 425: 434-438.

48. Monaghan AS, Mintenig GM, Sepúlveda FV. Outwardly rectifying $\mathrm{Cl}^{-}$channel in guinea pig small intestinal villus enterocytes: effect of inhibitors. Am J Physiol 1997; 273: G1141-G1152.

49. Mignen O, Egee S, Liberge M, Harvey BJ. Basolateral outward rectifier chloride channel in isolated crypts of mouse colon. Am J Physiol Gastrointest Liver Physiol 2000; 279: G277-G287.

50. Li Y, Halm ST, Halm DR. Secretory activation of basolateral membrane $\mathrm{Cl}^{-}$channels in guinea pig distal colonic crypts. Am J Physiol 2003; 284: C918-C933.

51. Kramer J, Hawley RS. The spindle-associated transmembrane protein Axs identifies a new family of transmembrane proteins in eukaryotes. Cell Cycle 2003; 2: 174-176.

52. Grubb BR, Vick RN, Boucher RC. Hyperabsorption of $\mathrm{Na}^{+}$ and raised $\mathrm{Ca}^{2+}$-mediated $\mathrm{Cl}^{-}$secretion in nasal epithelia of CF mice. Am J Physiol 1994; 266: C1478-C1483.

53. Anderson MP, Welsh MJ. Calcium and cAMP activate different chloride channels in the apical membrane of normal and cystic fibrosis epithelia. Proc Natl Acad Sci U S A 1991; 88: 6003-6007.

54. Boucher RC, Cheng EH, Paradiso AM, Stutts MJ, Knowles MR, Earp HS. Chloride secretory response of cystic fibrosis human airway epithelia. Preservation of calcium but not protein kinase C- and A-dependent mechanisms. J Clin Invest 1989; 84: 1424-1431.

55. Carles A, Millon R, Cromer A, Ganguli G, Lemaire F, Young $J$, et al. Head and neck squamous cell carcinoma transcriptome analysis by comprehensive validated differential display. Oncogene 2006; 25: 1821-1831.

56. Huang X, Godfrey TE, Gooding WE, McCarty KS Jr, Gollin 
SM. Comprehensive genome and transcriptome analysis of the 11q13 amplicon in human oral cancer and synteny to the 7F5 amplicon in murine oral carcinoma. Genes Chromosomes Cancer 2006; 45: 1058-1069.

57. Espinosa I, Lee CH, Kim MK, Rouse BT, Subramanian S, Montgomery $\mathrm{K}$, et al. A novel monoclonal antibody against DOG1 is a sensitive and specific marker for gastrointestinal stromal tumors. Am J Surg Pathol 2008; 32: 210-218.

58. Snouwaert JN, Brigman KK, Latour AM, Malouf NN, Boucher RC, Smithies O, et al. An animal model for cystic fibrosis made by gene targeting. Science 1992; 257: 1083-1088.

59. Cid LP, Niemeyer MI, Ramírez A, Sepúlveda FV. Splice variants of a CIC-2 chloride channel with differing functional characteristics. Am J Physiol 2000; 279: C1198-C1210. 